\title{
Hadron-argon cross-section measurements in ProtoDUNE-SP
}

\author{
Heng-Ye Liao ${ }^{1, *}$ \\ Department of Physics, Kansas State University, \\ 116 Cardwell Hall, 1228 N 17th St, Manhattan, KS 66506, USA \\ E-mail: liao@phys.ksu.edu
}

\begin{abstract}
The single-phase liquid argon ProtoDUNE detector (ProtoDUNE-SP) is located at CERN's neutrino platform facility and serves as a prototype to validate the technology for the 10-kton fiducial mass liquid argon detectors for the DUNE experiment. ProtoDUNE-SP was exposed to a variety of test-beam particles (electrons, muons, pions, kaons, and protons) towards the end of 2018 collecting data in a broad range of momenta, from $0.3-7 \mathrm{GeV} / \mathrm{c}$. The results will improve the understanding of final-state interactions in neutrino-argon cross-sections. The progress of the analyses towards the cross-section measurements on argon from the test-beam data collected at protoDUNE-SP will be presented.
\end{abstract}

40th International Conference on High Energy physics - ICHEP2020

July 28 - August 6, 2020

Prague, Czech Republic (virtual meeting)

${ }^{1}$ for the DUNE Collaboration.

${ }^{*}$ Speaker 


\section{Introduction}

Modern neutrino experiments use complex nuclei as neutrino targets. Hadrons produced in a neutrino interaction can re-interact with the nuclear medium before exiting the nucleus. This process is known as final state interactions (FSI). FSI can change charge, multiplicity of outgoing hadrons, and their final state kinematics. Primary neutrino interactions in the neutrino measurements can potentially be misinterpreted due to FSI. FSI are also a key component of the nuclear models (neutrino event generators) that neutrino experiments heavily rely on to unfold the reconstructed neutrino energy to the true neutrino energy. However, very limited measurements are available to validate these nuclear models.

The main physics goal of ProtoDUNE single phase (ProtoDUNE-SP) is to measure hadronargon cross-sections. Results provide critical information on hadron scattering in liquid argon and improve understanding of FSI in neutrino-argon interactions. Improved FSI models help to reduce systematic uncertainties on the neutrino energy reconstruction and neutrino signal selection.

\section{ProtoDUNE-SP at CERN Neutrino Platform}

Liquid argon time projection chambers (LArTPC) provide excellent tracking and calorimetric capabilities, enabling us to study neutrino-nucleus interactions with unprecedented precision. ProtoDUNE-SP utilizes the CERN H4 beamline with known particle types (pions, protons, kaons, muons, and electrons) and incident momenta $(0.3-7 \mathrm{GeV} / \mathrm{c})$, providing a controlled environment for better measurements of the particle interactions taking place within a LArTPC detector. A large dataset, $\sim 4.2$ million beam events, has been collected. The first results on ProtoDUNE-SP's detector performance that successfully meet the DUNE's required specifications have been published [1].

\section{Hadron-argon cross-section measurements in ProtoDUNE-SP}

Our major focus is on the proton inclusive cross-section, along with the pion inclusive and exclusive cross-sections. The inclusive cross-section of the pion and proton interaction consists of elastic and inelastic scattering channels ${ }^{2}$. The elastic cross-section means the argon nucleus is left in its ground state; the inelastic one refers to when the argon nucleus being left in an excited state and/or one or more nucleons are knocked out.

The pion exclusive cross-section consists of the charge exchange (CEx) and the absorption (Abs) interaction. Figure 1(a) shows a candidate event in pion CEx channel. The pion CEx refers to a single charge exchange that the final state pion charge differs by one unit from that of initial one $\left(\pi^{+} \rightarrow \pi^{0}\right)$. The event feature of the CEx channel is clearly visible: a final state $\pi^{0}$ decays and two photon showers are produced $\left(\pi^{0} \rightarrow \gamma \gamma\right)$. The signature of the pion Abs channel is that no pion appears in the final state. A candidate event for the pion Abs channel is shown in Figure 1(b). For the proton-argon interactions, a candidate event with inelastic scattering is displayed in Figure 1(c); another candidate event with elastic scattering is shown in Figure 1(d).

\footnotetext{
${ }^{2}$ Inelastic scattering interaction also called reactive interaction.
} 


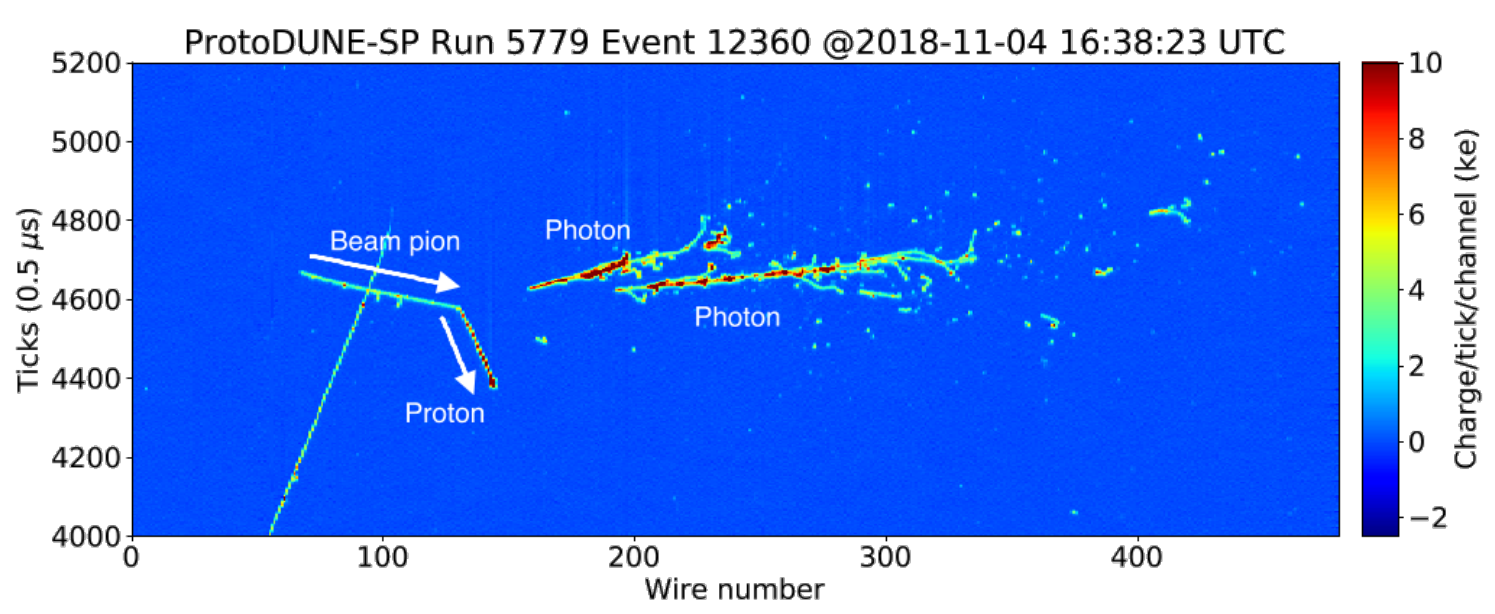

(a)

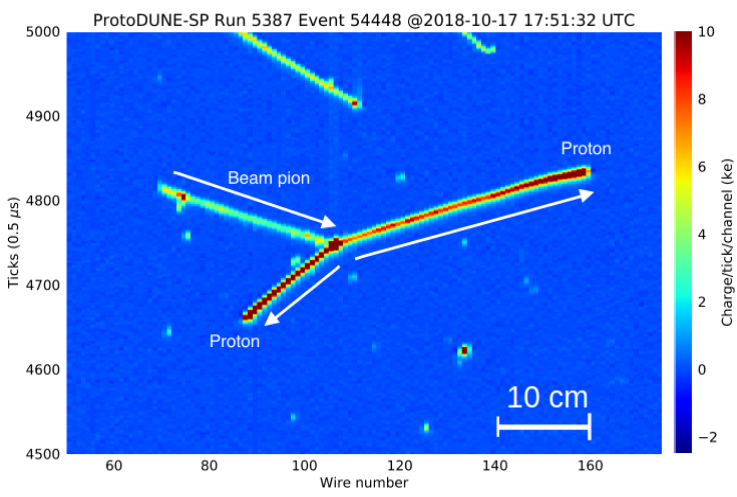

(b)

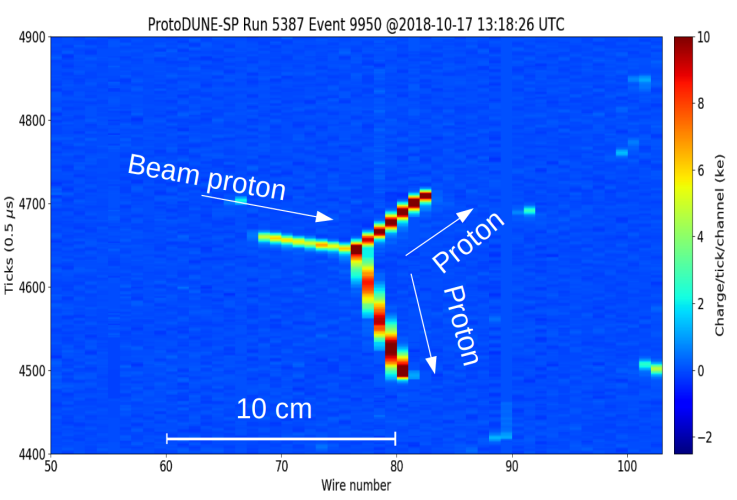

(c)

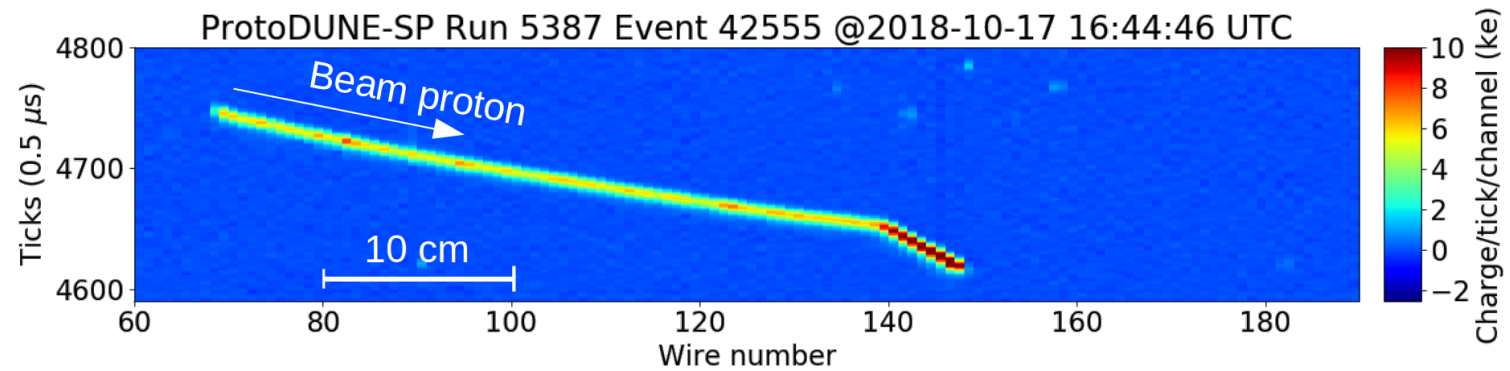

(d)

Figure 1: Candidate events of several pion and the proton interactions in ProtoDUNE-SP data: the pion charge exchange interaction (a), the pion absorption interaction (b), the proton inelastic scattering interaction (c), and the proton elastic scattering interaction (d).

\subsection{Cross-section determination methods}

ProtoDUNE-SP has developed two techniques to measure hadron-argon cross-sections. The first method is the thin-slice method, initially developed by the Liquid Argon In A Testbeam (LArIAT) Experiment (LArIAT) [2]. The thin-slice method uses wire-to-wire spacing as a series of "thin-slab" targets and each thin-slab is considered as an independent cross-section measurement. The cross-section $(\sigma)$ as a function of hadron kinetic energy (KE) is defined as $\sigma(K E)=S_{f}$. $R(K E)=S_{f} \cdot N(K E)^{\text {interacting }} / N(K E)^{\text {incident }}$, where $S_{f}$ is the scaling factor determined by 
the slab thickness and the target density, and its value is $~ 100$ barn for ProtoDUNE-SP (the slab thickness is $\sim 0.5 \mathrm{~cm}$ ). $N^{\text {interacting }}$ is the number of particles interacting in the slab and $N^{\text {incident }}$ is the number of particles impinging on the slab. The parameter $R(K E)$ describes the profile of the cross-section spectrum.

An alternative approach is the cross-section reweighting technique. ProtoDUNE-SP uses a software framework of cross-section reweighting based on Geant4, Geant4Reweight [3], to reweight the hadron-Ar cross-sections. The measured observables are served as representations of the crosssection model(s). The hadron-argon cross-section models can hence be validated by comparing the data observables with those of MC. Other non-cross-section-related issues that might affect the profiles of the observables need to be carefully addressed and measured. ProtoDUNE-SP has started investigating detector systematics and their impacts on these cross-section measurements.

\subsection{Hadron-argon cross-section analysis}

\subsubsection{Pion interactions}

Before the pions enter the LArTPC, they can be unambiguously identified using their timeof-flight and Cherenkov counter information from beamline instrumentation. After the particles enter the LArTPC, ProtoDUNE-SP uses the Pandora reconstruction package [4] for automated event reconstruction. The general feature of the pion CEx and the Abs channel is that no charged pion enters the final state. The event selection consists of two steps: (1) separating daughter particles into tracks and showers using a convolutional neural network (CNN), and (2) rejection of $\pi^{ \pm}$daughter tracks using a calorimetric-based particle identification (PID) method. Figure 2(a) shows the CNN track-like score distributions. The score value is between 0 (shower-like) and 1 (track-like). The track-like daughter particles are selected by applying the score value $\geq 0.34$. Figure 2(b) demonstrates the PID performance with a $\chi^{2}$ parameter. Clear separation between pion and proton daughters is observed. Rejection of $\pi^{ \pm}$daughter tracks is performed by placing the $\chi^{2}$ value greater than 50 .

To identify the pion CEx and Abs channels in advance, we search for $\pi^{0}$-like showers: the Abs channel has no showers while the CEx channel has showers in the final state. Figure 3(a) shows the distance between the primary reconstructed track end and the reconstructed shower start point. A CEx candidate is selected by setting the distance greater than $2 \mathrm{~cm}$. A similar cut is applied to select the $\pi^{0}$ showers using the number of hits for the reconstructed showers, as shown in Figure 3(b). The primary results of the performance of event selections are summarized in Table 1. The event selection performance achieved for the pion CEx and Abs channels are $67 \%$ efficiency and $66 \%$ purity; optimizing event selection for various pion interaction channels are in development.

Table 1: PID performance of the pion exclusive channel.

\begin{tabular}{ccc}
\hline Interaction Channel & Efficiency & Purity \\
\hline $\mathrm{CEx}+\mathrm{Abs}$ & $67 \%$ & $66 \%$ \\
$\mathrm{CEx}$ & $63 \%$ & $61 \%$ \\
$\mathrm{Abs}$ & $59 \%$ & $59 \%$ \\
\hline
\end{tabular}




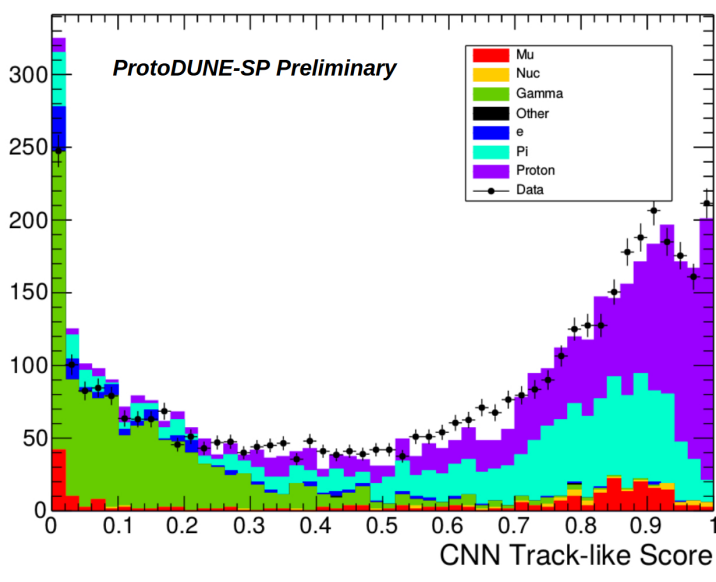

(a)

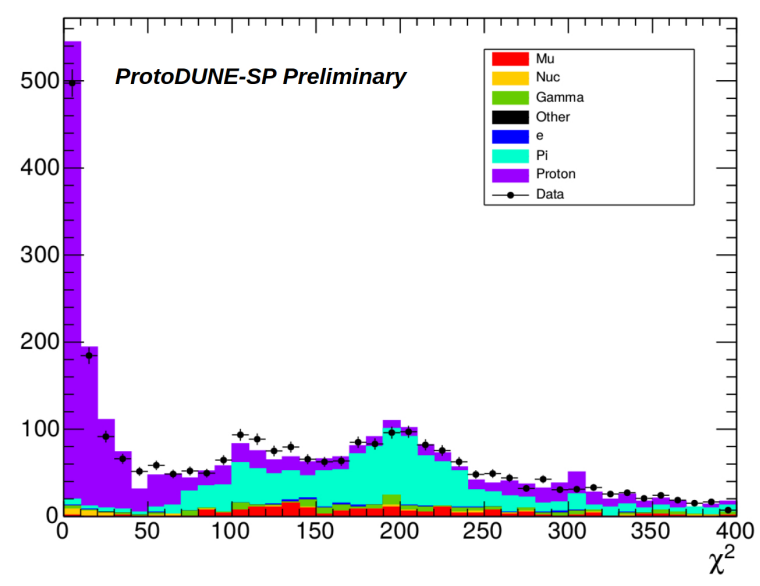

(b)

Figure 2: (a) The stacked CNN track-like score distributions of various daughter particles: black dots (data) and other colored histograms (MC). The score value is between 0 (shower-like) and 1 (track-like). (b) The stacked $\chi^{2}$ distributions of daughter particles. Separation between pions $\left(\pi^{ \pm}\right)$and protons is clearly visible.

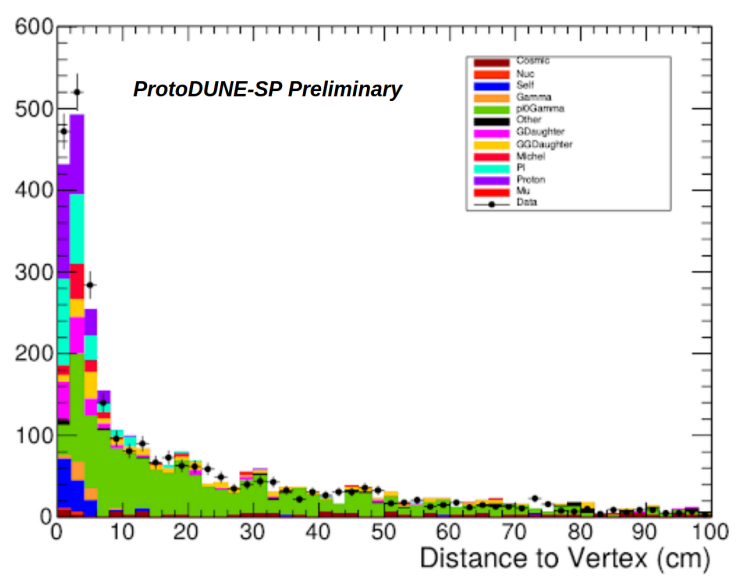

(a)

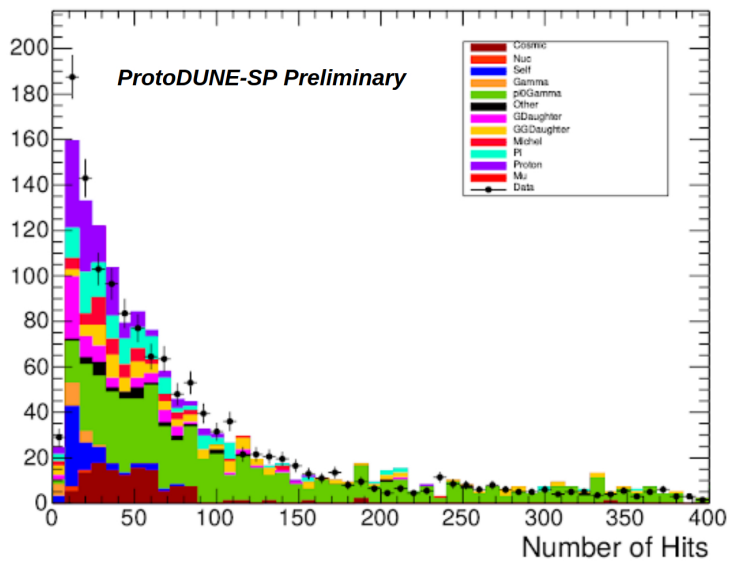

(b)

Figure 3: (a) The stacked distributions of distance between the primary reconstructed track end and the reconstructed shower start point for different daughter particles. (b) The stacked distributions of number of hits for the reconstructed showers.

\subsubsection{Proton interactions}

The normalized track length distribution is one of the key observables to deduce the protonargon cross-sections using the reweighting technique. Figure 4 shows the distributions of reconstructed proton track lengths, divided by their expected CSDA ranges ${ }^{3}$, the normalized track lengths for both data and MC. The distribution peak at 0.88 is dominated by stopping protons; the peak position is less than one because of the energy losses upstream and the space charge correction. The low tail is dominated by the protons with inelastic scattering, as their drift distances inside the LArTPC are shorter than those of stopping protons. This preliminary result shows reasonable

\footnotetext{
${ }^{3}$ Continuous slowing-down approximation (CSDA) range: average path length traveled by a charged particle as it slows down to rest.
} 
agreement between data and MC ( $\chi^{2} /$ ndf: 93.4/40). Addressing and evaluating detector systematics of the proton-argon cross-section measurements are ongoing efforts.

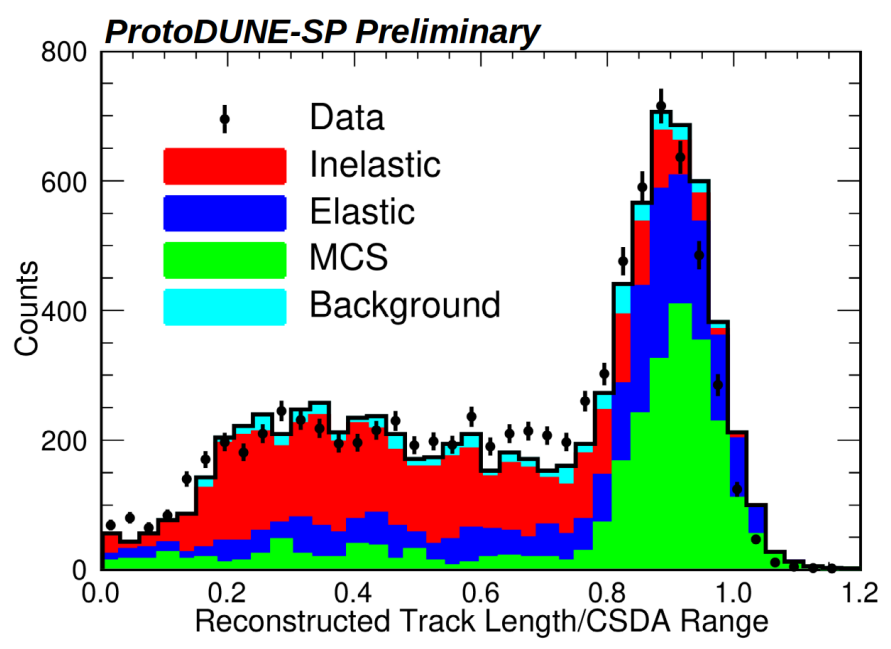

Figure 4: Distributions of reconstructed proton track lengths divided by the expected CSDA ranges. The incident beam momentum is $1 \mathrm{GeV} / c$. Data (black dots), MC with inelastic scattering (red), elastic scattering (blue), multiple Coulomb scattering, MCS (green), background (light blue) with protons having interactions outside the LArTPC or being reconstructed as the wrong particles, and the sum of all MC components (black histogram).

\section{Summary}

ProtoDUNE-SP measures the hadron-argon cross-sections in a controlled environment, providing valuable inputs for better understandings of FSI for neutrino-argon interactions. The crosssection determination methods for the measurement of hadron-argon cross-sections are developed. Rapid progress has been made on both pion-argon and proton-argon cross-section analyses. The preliminary results of the pion event selections for the charge exchange and the absorption channels are presented. The observable used for the proton-argon cross-section reweighting is demonstrated.

\section{References}

[1] Dune Collaboration, "First results on ProtoDUNE-SP liquid argon time projection chamber performance from a beam test at the CERN Neutrino Platform", arXiv: 2007.06722 (2020) (link)

[2] LARIat Collaboration, "The Liquid Argon In A Testbeam (LArIAT) experiment", JINST 15 P04026 (2020) (link)

[3] J. Calcutt, C. Thorpe, K. Mahn, L. Fields, "Geant4Reweight: a framework for reweighting and evaluating systematics related to hadron interactions in GEANT4", paper in preparation.

[4] Microboone Collaboration, "The Pandora multi-algorithm approach to automated pattern recognition of cosmic-ray muon and neutrino events in the MicroBooNE detector", EPJC vol. 78, 82 (2018) (link) 